\title{
Global Conservation Laws and Femtoscopy of Small Systems
}

\author{
Zbigniew Chajęcki ${ }^{1}$ and Mike Lisa ${ }^{1}$ \\ ${ }^{1}$ Department of Physics, Ohio State University, \\ 1040 Physics Research Building, \\ 191 West Woodruff Ave, Columbus, OH 43210, USA
}

\section{Received on 16 December, 2006}

\begin{abstract}
It is increasingly important to understand, in detail, two-pion correlations measured in $p+p$ and $d+A$ collisions. In particular, one wishes to understand the femtoscopic correlations, in order to compare to similar measurements in heavy ion collisions. However, in the low-multiplicity final states of these systems, global conservation laws generate significant $N$-body correlations which project onto the two-pion space in non-trivial ways and complicate the femtoscopic analysis. We discuss a model-independent formalism to calculate and account for these correlations in measurements.
\end{abstract}

Keywords: Proton collisions; Femtoscopy; Heavy ions; Pion correlations; RHIC; LHC

\section{INTRODUCTION}

The unique and distinguishing feature of heavy ions is their large (relative to the confinement scale) size and the possibility to generate bulk systems which may be described in thermodynamic terms, allowing to discuss the Equation of State of strongly-interacting matter. The primary evidence for the creation of bulk matter at the highest energies is the existence of strong collective flow [1]. The dominant feature of flow are the correlations between space and momentum which it generates; thus, momentum-only observables such as $p_{T}$ spectra and azimuthal anisotropies [2-5] represent only an indirect projection of the effect. Femtoscopic measurements access space as a function of particle momentum, thus providing the most direct probe of the most crucial feature of heavy ion collisions [c.f. e.g. 6]. In particular, flow is manifest by a negative correlation between the "HBT radius" and the transverse mass $\left(m_{T}\right)$ of the particles [7].

Clearly, then, a detailed understanding of femtoscopic measurements in heavy ion collisions is crucial to proving the existence of, or probing the nature of, the bulk system generated in the collision. It is in fact possible to quantitatively interpret both the femtoscopic and momentum-only observations at RHIC in consistent, flow-dominated models of the system [e.g. 8]. All seems well.

However, two-pion femtoscopic measurements are also common in $e^{+}+e^{-}$or $p+p(\bar{p})$ collisions [9]. In these collisions, too, "HBT radii" are observed to fall with $m_{T}$. Speculations of the physics behind this observation have included Heisenberg uncertainty-based arguments, stringbreaking phenomena, and temperature gradients; an excellent overview may be found in [10]. Typically, however, one might not expect the system created in a $p+p$ collision to exhibit bulk behavior similar to that from heavy ion collisions.

Quantitative comparisons between femtoscopic measurements in $A+A$ and $p+p$ systems have been complicated because techniques for event-mixing, frame definitions, and the like, have been different in the particle-physics and heavy-ion communities. As importantly, kinematic acceptance and collision energies are usually quite different. Recently, however, the STAR experiment has reported the first preliminary study of directly-comparable femtoscopic measurements from $A+A$ and $p+p$ systems [11] at the same $\sqrt{s_{N N}}$, using the same detector, and with identical techniques. The results indicate that the femtoscopic probe of flow- falling "HBT radii" with $m_{T}-$ is essentially identical in the small and large systems. This might signal an unexpected "universality" in the spatial substructure of hadronic and heavy ion collisions. Unravelling the physics behind this similarity might provide new insight into the dynamical space-time substructure of both hadronic and heavy ion collisions.

Before drawing strong physics conclusions from "HBT radii" coming from fits to the pion correlations measured in $p+p$ collisions, however, the measured correlation functions themselves must be understood in detail. The STAR data show clear non-femtoscopic correlations which must be disentangled from the femtoscopic ones [11]. Femtoscopic correlations are those which depend directly on the two-particle separation distribution [c.f. 6]. Non-femtoscopic correlations may arise from string or jet fragmentation, resonance decay, or global conservation laws.

In this work, we explore the projection of $N$-body Energy and Momentum Conservation-Induced Correlations (EMCICs) onto a two-particle relative momentum correlation function. In Section II we briefly discuss the harmonic representation of the correlations which best illustrates the effect. In Section III we discuss EMCICs generated by a Monte Carlo event generator containing only global conservation laws. A method to calculate analytically (but using distributions from the data) EMCICs is shown in Section IV. This provides an "experimentalist's formula," given in Section V, useful to disentangle EMCICs from the data, allowing a femtoscopic analysis of the correlation functions. We summarize in Section VI.

\section{SPHERICAL HARMONIC DECOMPOSITION OF CORRELATION FUNCTIONS}

At asymptotically high relative momentum $|\vec{q}|$ (or $\left|\overrightarrow{k^{*}}\right|$ ), femtoscopic contributions to the the correlation function (those described by the Koonin-Pratt equation [discussed in 


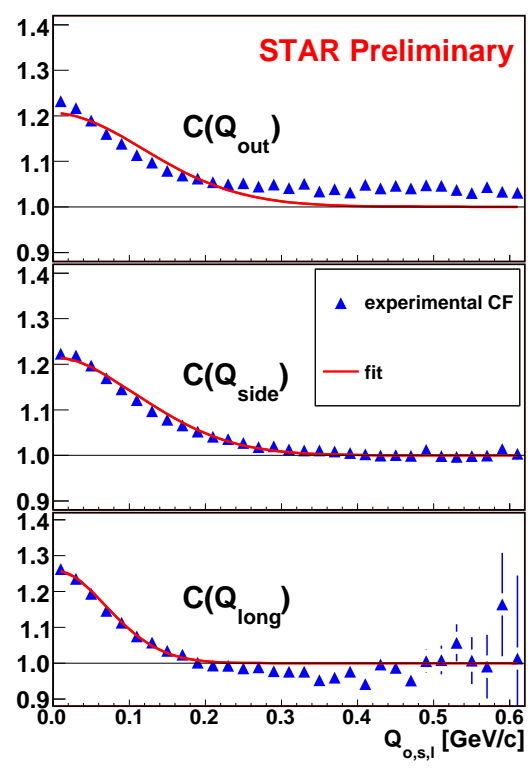

FIG. 1: (Color online) Preliminary STAR two-pion correlation functions [11] presented as 1D projections in the Bertsch-Pratt decomposition.

6]) must approach a constant value, usually normalized to unity, independent of the direction of $\vec{q}$. Preliminary STAR measurements [11] of small systems, Fig. 1, show clear nonfemtoscopic correlations in addition. Also shown is a fit with the commonly Gaussian (with Coulomb suppression) functional form [6]. Clearly, the fit is a poor representation of the data. We stress, however, that it is not the (non-)Gaussian nature of the source at issue here; any source function will lead to vanishing femtoscopic correlations at large $|\vec{q}|$ and will thus contradict the data.

We further stress that the problem is not one of normalization. Shown in the Figure is the common representation of the 3-dimensional correlation function into three 1-dimensional axes [cf 6]. The projections, then, are not independent and cannot be independently normalized. The problem is that the value approached at large $|\vec{q}|$ depends on the direction in $\vec{q}$ space.

One-dimensional projections present a limited tool for exploring detailed structure of the three-dimensional correlation function. The spherical harmonic decomposition (SHD) is a much more efficient representation of the data which uses all of the data to show the shape of the correlation function in 3D $\vec{q}$ space. There, the spherical harmonic coefficients $A_{l, m}$, which depend on $Q \equiv|\vec{q}|$, are calculated as

$$
\begin{aligned}
A_{l, m}(Q)=\sum_{\text {bins } i} C\left(Q, \cos \theta_{i}, \phi_{i}\right) . & \\
& Y_{l, m}\left(\cos \theta_{i}, \phi_{i}\right) F_{l, m}\left(\cos \theta_{i}, \Delta_{\cos \theta}, \Delta_{\phi}\right),
\end{aligned}
$$

where $F_{l, m}$ represents a numerical factor correcting for finite bin sizes $\Delta_{\cos \theta}$ and $\Delta_{\phi}$; it turns out not to depend on $\phi_{i}$. The angles $\theta$ and $\phi$ are related to the Bertsch-Pratt Cartesian coordinate system through

$$
q_{o}=Q \sin \theta \cos \phi, \quad q_{s}=Q \sin \theta \sin \phi, \quad q_{l}=Q \cos \theta .
$$
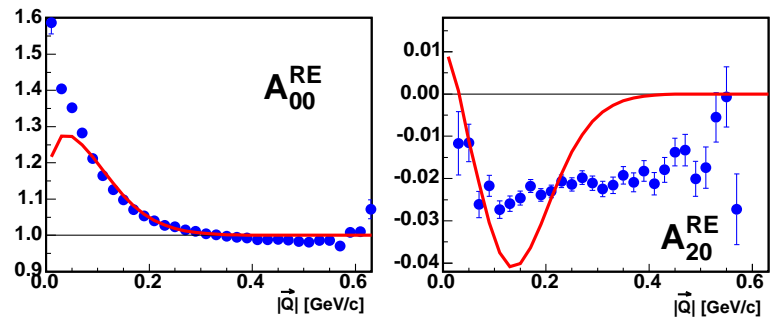

\section{STAR PRELIMINARY}

- peripheral d+Au @ $200 \mathrm{GeV}$
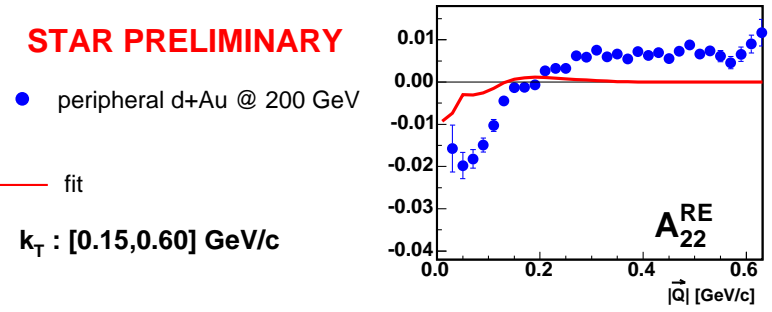

FIG. 2: (Color online) Preliminary STAR two-pion correlation functions [11] presented in the SHD representation.

See [12] for a complete discussion.

Preliminary STAR correlation functions in the SHD representation [11] are shown in Fig. 2. Coefficients for $l \geq 4$ are much less significant, compared to error bars; to good approximation, the non-femtoscopic behavior is quadrupole $(l=2)$ in nature.

The presence of non-femtoscopic correlations is clear from the non-vanishing behavior of $A_{l \neq 0, m}$ 's at large $Q$. However, it is by no means clear that these contributions to the correlation function are confined to large $Q$. Thus, one cannot attempt to interpret the low- $Q$ region only in terms of femtoscopic correlations, while parameterizing or ignoring the large- $Q$ region; see [13] for further discussion.

\section{EMCICS GENERATED BY THE GENBOD MONTE CARLO GENERATOR}

Non-femtoscopic correlations may arise from a variety of sources. Jets will clearly induce momentum-space correlations between its fragmentation products. While this cannot be discounted, the low momentum of the pions under consideration $\left(p_{T} \sim 0.2 \mathrm{GeV}\right)$ puts us squarely in the region in which factorization breaks down and the jet interpretation becomes significantly murkier. We do not explore this possibility here. In the kinematic region under consideration, string fragmentation may play a role; this is an area for future study, though significant model-dependence will be present.

Resonances induce correlations among daughters as well; while these might even dominate $\pi^{+} \pi^{-}$correlations, they should be negligible for identical pion correlations. Collective bulk flow (e.g. anisotropic elliptic flow) will generate $N$ body correlations which will project onto the two-body space. Non-femtoscopic correlations of the type observed by STAR in small systems are not, however, observed in Au+Au collisions, despite the fact that elliptic flow is much larger there; therefore, we do not believe that collective flow generates the observed effects. 


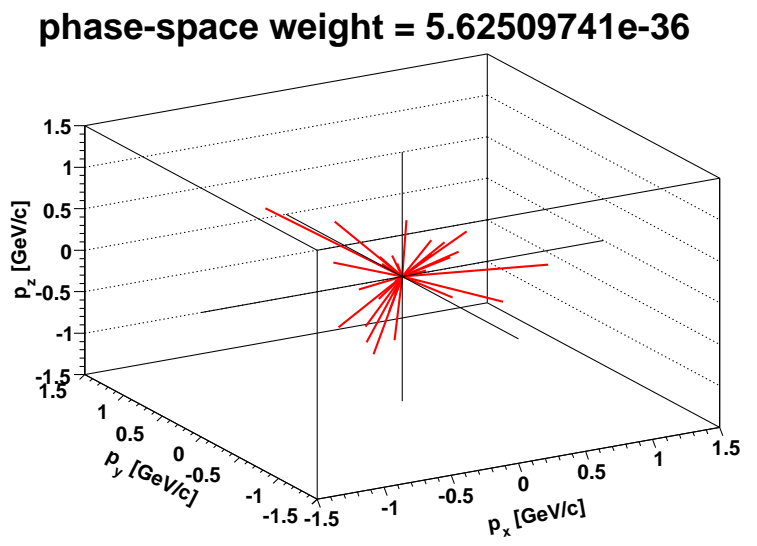

FIG. 3: (Color online) A high-probability multiplicity-30 event calculated by GENBOD . Lines correspond to particle momenta $p_{x}, p_{y}, p_{z}$

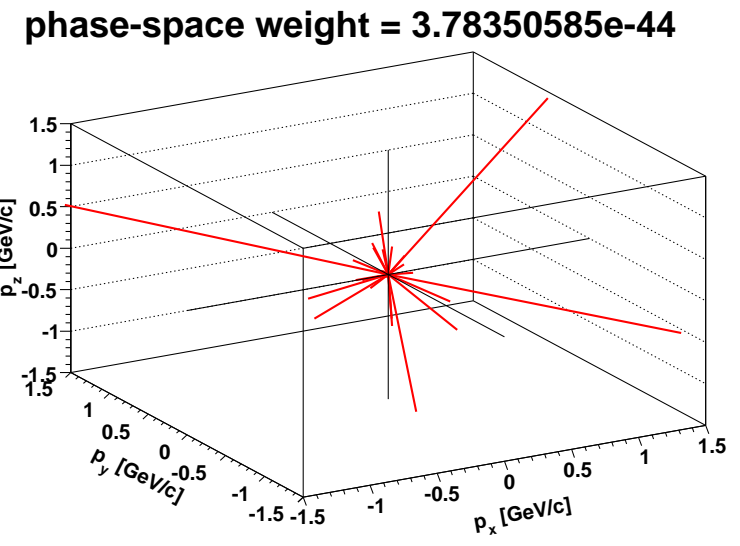

FIG. 4: (Color online) A low-probability multiplicity-30 event calculated by GENBOD . Lines correspond to particle momenta $p_{x}, p_{y}, p_{z}$.

Without doubt, one physical effect which must be at play is momentum and energy conservation. As global conservation laws, these provide an $N$-body constraint on the event, which projects down onto 2-body spaces. The observed nonfemtoscopic effects [11] become more and more significant as the multiplicity $(N)$ of the event decreases, as expected from conservation laws. It is these EMCICs which we focus on here.

To clearly understand the role of EMCICs, we would like to have events in which there is no other physics involved besides the conservation laws. Such a tool has been provided almost 40 years ago in the form of the GENBOD computer program [see 14, for an excellent write-up of the method and physics] in the CERN library. Given a requested total multiplicity $(N)$, a list of masses $\left(m_{i}\right)$ of emitted particles, and a total amount of energy $\left(E_{\text {tot }}\right)$ to distribute among them, GENBOD returns an event of random momenta (four vectors $p_{j}$ ), subject only to the condition of energy and momentum conservation. More importantly, it returns, for each event, a weight proportional to the probability that the event will actually oc-
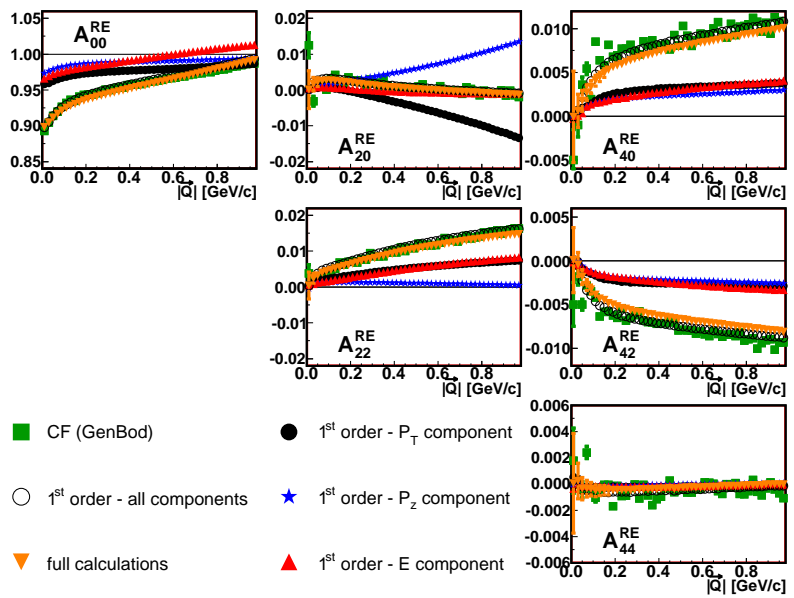

FIG. 5: (Color online) SHD coefficients for GENBOD -generated events consisting of 18 pions, as measured in the pair CMS frame. Green squares are $A_{l, m}$ 's from the GENBOD events. For discussion of the other symbols, see Section IV.
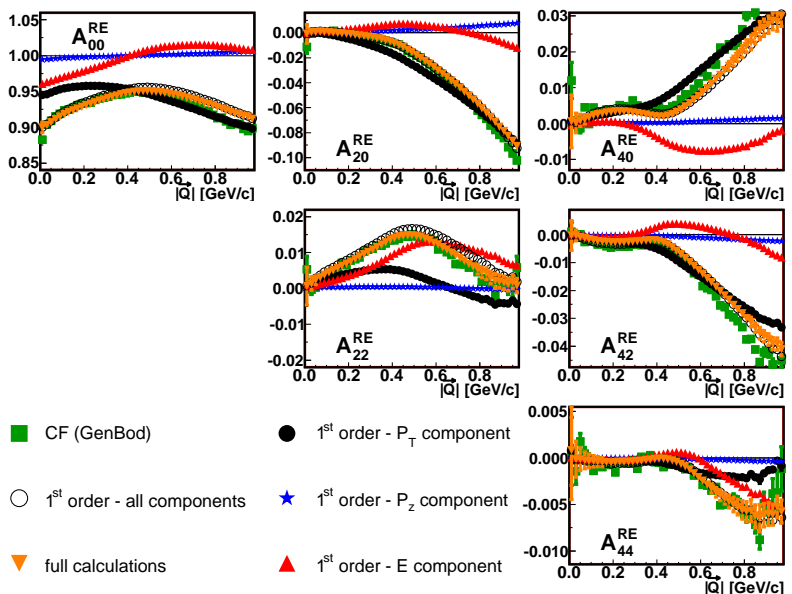

FIG. 6: (Color online) Same as in Fig. 5, except only using pions with $|\eta|<0.5$ in the correlation function.

cur in nature. This weight is proportional to the phasespace integral $R_{N}$

$$
R_{N}=\int^{4 N} \delta^{4}\left(P-\sum_{j=1}^{N} p_{j}\right) \prod_{i=1}^{N} \delta\left(p_{i}^{2}-m_{i}^{2}\right) d^{4} p_{i},
$$

where $P=\left(E_{\mathrm{tot}}, \overrightarrow{0}\right)$ is the total momentum four-vector of the event. See [14] for a practical iterative prescription to calculate $R_{N}$. Thus, it is a much different tool than, say RQMD, in which each event returned may be treated as equally probable.

We select (via Monte Carlo) GENBOD events according to their weight and run them through identical software as used for experimental analysis. Fortunately, the code is fast, since one must calculate large statistics from which to select. This is because the phase-space weights vary by large factors. As a very extreme case, Figs. 3 and 4 show a likely and unlikely event, respectively, for multiplicity $N=30$. As one would 

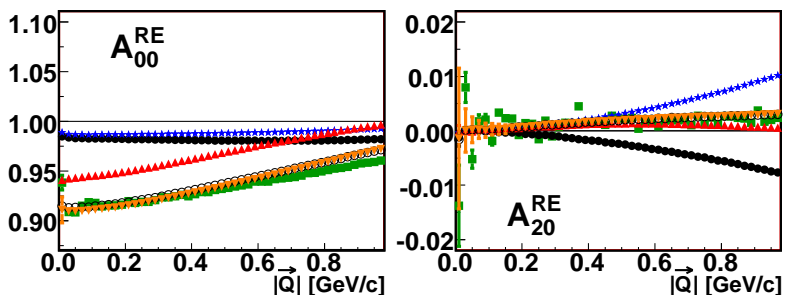

- CF (GenBod)

- $1^{\text {st }}$ order $-\mathrm{P}_{\mathrm{T}}$ component

$\star \quad 1^{\text {st }}$ order $-\mathrm{P}_{\mathrm{z}}$ component

- $1^{\text {st }}$ order - E component

$\bigcirc \quad 1^{\text {st }}$ order - all components

$\nabla$ full calculations

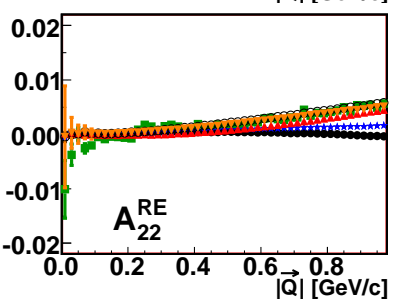

FIG. 7: (Color online) SHD coefficients for GENBOD -generated events consisting of 18 pions, as measured in the pair LCMS frame. Green squares are $A_{l, m}$ 's from the GENBOD events. For discussion of the other symbols, see Section IV.
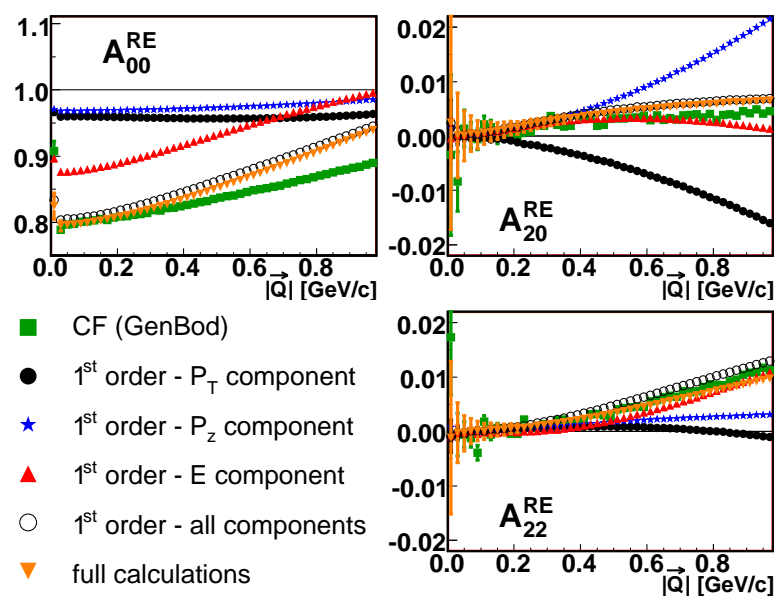

FIG. 8: (Color online) Same as Fig. 7, but for 9-pion events.

expect, the "rounder" event is more likely, though one might be surprised by the factor of a hundred million between the probabilities.

Figures 5 and 6 show the $A_{l, m}$ 's calculated by GENBOD for 18 -pion events without and with a selection of $|\eta|<0.5$, respectively. Note that this cut applies to the pions which are used in the analysis, not to the set of particles for which energy and momentum is conserved; energy and momentum is always conserved for the full event. Clearly visible are significant and nontrivial $A_{l, m}$ 's due only to EMCICs. We observe also that the $l=4$ coefficients are about an order of magnitude smaller than the $l=2$ ones; this is generically expected [cf 12]. Comparing the two figures, it is clear that kinematical selection has significant effect on the EMCIC effects. Also significant (but not shown) is whether one includes other species (say protons) into the mix of emitted particles.

Comparison of Figs. 7, 8 and 9 makes clear the multiplicity dependence of the EMCICs. As expected, lower multiplicity events show a greater effect. Also (not shown), increasing the
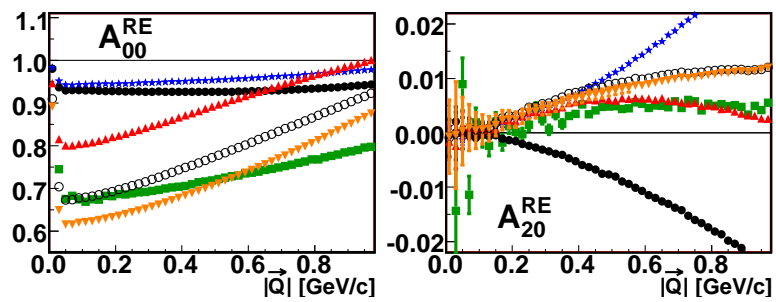

$\begin{array}{ll}\text { - } & \text { CF (GenBod) } \\ \text { - } & 1^{\text {st }} \text { order }-\mathrm{P}_{\mathrm{T}} \text { component }\end{array}$

$\star \quad 1^{\text {st }}$ order $-\mathrm{P}_{\mathrm{z}}$ component

$\Delta \quad 1^{\text {st }}$ order - E component

$\bigcirc 1^{\text {st }}$ order - all components

$\nabla$ full calculations

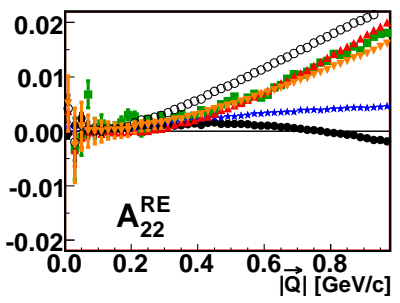

FIG. 9: (Color online) Same as Fig. 7, but for 6-pion events.

amount of energy to be distributed among the particles, for fixed multiplicity, decreases EMCICs, as one expects.

Finally, we note that EMCICs can affect the correlation function even down to very low $Q$, again reminding us that we cannot (responsibly) ignore these effects in a femtoscopic analysis.

\section{ANALYTIC CALCULATION OF EMCICS}

Now then, EMCIC effects generated by GENBOD "resemble" the experimental data, but it is likely unwise to use GENBOD itself to correct the data for several reasons. Firstly, there is strong sensitivity to the (not completely measured) number and species-mix of all particles emitted in the event, including neutrinos and possible magnetic monopoles (or, less exotically, particles escaping detector acceptance). Secondly, there is strong sensitivity to the energy "available" in the event; it is not obvious that this is $\sqrt{s_{N N}}$ of the collision. Clearly, EMCIC effects depend on the individual momenta $\vec{p}_{1}$ and $\vec{p}_{2}$ of the particles entering the correlation function. This will depend on acceptance, efficiency, kinematic cuts and, to a degree, the underlying single-particle phasespace. (While correlation functions are insensitive to the single-particle phasespace, the correlations which they measure may, in fact, depend on this phasespace, due to physical effects.)

Thus, one would like to calculate EMCICs, based on the data itself. Here, we expand upon previous results [15-17] to write down correction factors which implement EMCICs onto multi-particle distributions.

\section{A. Integral correction factors}

Danielewicz [15], and later Borghini, Dinh and Ollitraut [16], considered EMCIC-type effects on two-particle azimuthal correlations (elliptic flow $v_{2}$ ). They considered transverse momentum $\left(\vec{P}_{T}\right)$ conservation only, but Borghini later [17] generalized to the case of an arbitrary number $D$ of independent (orthogonal) spatial dimensions and very re- 
cently considered momentum conservation effects on threeparticle analyses of jet-like behavior [18].

As we shall see below, the conservation of energy generates effects of similar magnitude as does conservation of (three-) momentum. We deal only with on-shell particles, for which energy cannot be treated as independent of the momentum (as, say, $p_{x}$ would be largely independent of $p_{y}$ ). Thus, it is far from clear that we may simply take the $D=4$ case of reference [17]. In fact, the distinction is significant in the final result, since in $D=3 \oplus 1$, conservation of the final component couples into the first three, unlike the $D=4$ case [20].

Here, we remain in the case of interest $-D=3$ spatial dimensions - and conserve 3-momentum $\vec{p}$. We implement energy conservation and on-shell constraints a bit later.

We define [21]

$$
f\left(\vec{p}_{i}\right) \equiv \frac{d^{3} N}{d \vec{p}_{i}^{3}}
$$

as the single-particle momentum distribution unaffected by EMCICs. Then, the $k$-particle distribution ( $k$ less than the total multiplicity $N$ ) including EMCICs is

$$
\begin{aligned}
f_{c}\left(\vec{p}_{1}, \ldots, \vec{p}_{k}\right)= & \left(\prod_{i=1}^{k} f\left(\vec{p}_{i}\right)\right) \times \\
& \frac{\int\left(\prod_{j=k+1}^{N} d^{3} \vec{p}_{j} f\left(\vec{p}_{j}\right)\right) \delta^{3}\left(\sum_{i=1}^{N} \vec{p}_{i}\right)}{\int\left(\prod_{j=1}^{N} d^{3} \vec{p}_{j} f\left(\vec{p}_{j}\right)\right) \delta^{3}\left(\sum_{i=1}^{N} \vec{p}_{i}\right)}
\end{aligned}
$$

(Note the difference between numerator and denominator in the starting value of the index $j$ on the product.)

In our case, we add in total energy conservation $\sum E_{i}=$ $\sqrt{s}$, which simply entails the replacement $\delta^{3}\left(\sum_{i} \vec{p}_{i=1}^{N} i\right) \rightarrow$ $\delta^{4}\left(\sum_{i=1}^{N} p_{i}-P\right)$ in Eq. 5, where $P=(\sqrt{s}, \overrightarrow{0})$ is the total energy-momentum of the event, and $p_{i}^{0}=E_{i}=\sqrt{\vec{p}_{i}^{2}+m_{i}^{2}}$ is the energy of the on-shell particle.

To apply the central limit theorem (below) to energy and momentum, we need to integrate independently over both. We begin by using the Lorentz-invariant distributions

$$
\tilde{f}\left(p_{i}\right) \equiv 2 E_{i} \frac{d^{3} N}{d \vec{p}_{i}^{3}}=2 E_{i} f\left(p_{i}\right)
$$

and simply rewriting Eq. 5 as

$$
\begin{aligned}
& \tilde{f}_{c}\left(p_{1}, \ldots, p_{k}\right)=\left(\prod_{i=1}^{k} \tilde{f}\left(p_{i}\right)\right) \times \\
& \frac{\int\left(\prod_{j=k+1}^{N} \frac{d^{3} \vec{p}_{j}}{E_{j}} \tilde{f}\left(\vec{p}_{j}\right)\right) \delta^{4}\left(\sum_{i=1}^{N} p_{i}-P\right)}{\int\left(\prod_{j=1}^{N} \frac{d^{3} \vec{p}_{j}}{E_{j}} \tilde{f}\left(\vec{p}_{j}\right)\right) \delta^{4}\left(\sum_{i=1}^{N} p_{i}-P\right)}
\end{aligned}
$$

$$
\begin{aligned}
= & \left(\prod_{i=1}^{k} \tilde{f}\left(p_{i}\right)\right) \times \\
& \frac{\int\left(\prod_{j=k+1}^{N} d^{4} p_{j} \delta\left(p_{j}^{2}-m_{j}^{2}\right) \tilde{f}\left(p_{j}\right)\right) \delta^{4}\left(\sum_{i=1}^{N} p_{i}-P\right)}{\int\left(\prod_{j=1}^{N} d^{4} p_{j} \delta\left(p_{j}^{2}-m_{j}^{2}\right) \tilde{f}\left(p_{j}\right)\right) \delta^{4}\left(\sum_{i=1}^{N} p_{i}-P\right)} \\
= & \left(\prod_{i=1}^{k} \tilde{f}\left(p_{i}\right)\right) \times \\
& \frac{\int\left(\prod_{j=k+1}^{N} d^{4} p_{j} g\left(p_{j}\right)\right) \delta^{4}\left(\sum_{i=1}^{N} p_{i}-P\right)}{\int\left(\prod_{j=1}^{N} d^{4} p_{j} g\left(p_{j}\right)\right) \delta^{4}\left(\sum_{i=1}^{N} p_{i}-P\right)} .
\end{aligned}
$$

Thus, we arrive at an integral over four independent variables, in which the integrand function $g(p)$ is "highly peaked" and with strong correlations in the 4-D $p$-space.

According to Eq. 7, the $k$-body momentum distribution, including EMCICs, is the $k$-body distribution not affected by EMCICs - i.e. just an uncorrelated product of single-particle distributions - multiplied by a "correction factor" which enforces the EMCIC. The numerator of this factor just demands that the remaining $N-k$ on-shell particles are configured so as to conserve total energy and momentum, and the denominator just normalizes the distribution.

\section{B. Application of the Central Limit Theorem}

To arrive at a useful result, we wish to use the arguments of [15-17] to apply the central limit theorem (CLT) to Eq. 7. Those authors note that the distribution of a large number $M$ of uncorrelated momenta $P^{\prime}=\sum_{i=1}^{M} p_{i}$ is, by the Central Limit Theorem, a Gaussian distribution

$$
\begin{aligned}
F_{M}\left(P^{\prime}\right) & \equiv \int\left(\prod_{i=1}^{M} d^{4} p_{i} g\left(p_{i}\right)\right) \delta^{4}\left(\sum_{i=1}^{M} p_{i}-P^{\prime}\right) \\
& =\frac{1}{\pi \sigma^{2}} \exp \left(-\frac{\left(P^{\prime}\right)^{2}}{2 \sigma^{2}}\right) .
\end{aligned}
$$

Strictly speaking, it is not obvious that application of the CLT is valid in our case. The authors of [15-17] neglect any correlation in $p$-space of the single-particle distribution $g(p)$. This means neglecting elliptic flow and any longitudinal dependence of the $p_{T}$ distribution. These approximations should be fine, since the authors consider small $v_{2}$, and discuss effects of transverse momentum only. In our cases, however, $g(p)$ obviously has very strong correlations in the $3+1$ space, due to the on-shell constraint. It turns out [22] however, that the correlation between the total momentum and total energy of a system of $N$ particles becomes quickly negligible for $N \gtrsim 10$. We then recognize the integral in the numerator in Eq. 7 as the distribution of $N-k$ momenta $\sum_{j=k+1}^{N} p_{j}=P-\sum_{j=1}^{k} p_{j}$ 
so that for "large enough" $N-k$, we find [23]

$$
\begin{gathered}
\tilde{f}_{c}\left(p_{1}, \ldots, p_{k}\right)=\left(\prod_{i=1}^{k} \tilde{f}\left(p_{i}\right)\right) \times \\
\left(\frac{N}{N-k}\right)^{2} \cdot \exp \left[-\sum_{\mu=0}^{3} \frac{\left(\sum_{i=1}^{k}\left(p_{i, \mu}-\left\langle p_{\mu}\right\rangle\right)\right)^{2}}{2(N-k) \sigma_{\mu}^{2}}\right]
\end{gathered}
$$

where

$$
\sigma_{\mu}^{2} \equiv\left\langle p_{\mu}^{2}\right\rangle-\left\langle p_{\mu}\right\rangle^{2}
$$

and

$$
\left\langle p_{\mu}^{2}\right\rangle \equiv \int d p \tilde{f}(p) \cdot p_{\mu}^{2}
$$

Naturally, $\left\langle p_{(\mu=1,2,3)}\right\rangle=0$. (In these equations, we now assume only one species of particles, so that no species label is needed for $\left\langle p_{\mu}^{2}\right\rangle$. This is only for simplicity of notation here; results, including the "experimentalist's formula" below, only become more cumbersome to write, but are similar otherwise.)

Note that even the single-particle momentum distribution is affected by EMCICs

$$
\begin{gathered}
\tilde{f}_{c}\left(p_{i}\right)=\tilde{f}\left(p_{i}\right) \cdot\left(\frac{N}{N-1}\right)^{2} \times \\
\exp \left[-\frac{1}{2(N-1)}\left(\frac{p_{i, x}^{2}}{\left\langle p_{x}^{2}\right\rangle}+\frac{p_{i, y}^{2}}{\left\langle p_{y}^{2}\right\rangle}+\frac{p_{i, z}^{2}}{\left\langle p_{z}^{2}\right\rangle}+\frac{\left(E_{i}-\langle E\rangle\right)^{2}}{\left\langle E^{2}\right\rangle-\langle E\rangle^{2}}\right)\right]
\end{gathered}
$$

The $k$-particle correlation function is defined as the measured (i.e. EMCIC-affected) $k$-particle yield divided by the product of the measured single-particle yields

$$
\begin{aligned}
& C\left(p_{1}, \ldots, p_{k}\right) \equiv \frac{\tilde{f}_{c}\left(p_{1}, \ldots, p_{k}\right)}{\tilde{f}_{c}\left(p_{1}\right) \cdots \tilde{f}_{c}\left(p_{k}\right)}=\frac{\left(\frac{N}{N-k}\right)^{2}}{\left(\frac{N}{N-1}\right)^{2 k}} \times \\
& \frac{\exp \left[\frac{-1}{2(N-k)}\left\{\sum_{\mu=1}^{3}\left(\frac{\left(\sum_{i=1}^{k} p_{i, \mu}^{2}\right)^{2}}{\left\langle p_{\mu}^{2}\right\rangle}\right)+\frac{\left(\sum_{1}^{k}\left(E_{i}-\langle E\rangle\right)\right)^{2}}{\left\langle E^{2}\right\rangle-\langle E\rangle^{2}}\right\}\right]}{\exp \left[\frac{-1}{2(N-1)} \sum_{i=1}^{k}\left\{\sum_{\mu=1}^{3} \frac{p_{i, \mu}^{2}}{\left\langle p_{\mu}^{2}\right\rangle}+\frac{\left(E_{i}-\langle E\rangle\right)^{2}}{\left\langle E^{2}\right\rangle-\langle E\rangle^{2}}\right\}\right]}
\end{aligned}
$$

An important point: EMCICs result from the constraint that the event's energy-momentum is the same fixed number for all pairs in the event. This is true in the laboratory frame, but not in LCMS or pair rest frame. Thus, while one may bin the correlation function in the frame of one's choice, the momenta which appear on the right-hand-side of Eq. 13 must be in the laboratory system.

To first order in $1 / N$, the two-particle correlation function becomes

$$
\begin{gathered}
C\left(p_{1}, p_{2}\right)=1- \\
\frac{1}{N}\left(2 \frac{\vec{p}_{1, T} \cdot \vec{p}_{2, T}}{\left\langle p_{T}^{2}\right\rangle}+\frac{p_{1, z} \cdot p_{2}, z}{\left\langle p_{z}^{2}\right\rangle}+\frac{\left(E_{1}-\langle E\rangle\right)\left(E_{2}-\langle E\rangle\right)}{\left\langle E^{2}\right\rangle-\langle E\rangle^{2}}\right)
\end{gathered}
$$

where we have taken $\left\langle p_{x}^{2}\right\rangle=\left\langle p_{y}^{2}\right\rangle=\left\langle p_{T}^{2}\right\rangle / 2$. In what follows, we shall refer to the first, second, and third terms within the parentheses of Eq. 14 as the " $p_{T}$ term," " $p_{z}$ term," and " $E$ term," respectively.

If we know $N,\left\langle p_{T}^{2}\right\rangle,\left\langle p_{z}^{2}\right\rangle,\left\langle E^{2}\right\rangle$, and $\langle E\rangle$ from the data, we can calculate EMCICs using Eq. 13. Better yet, if $N$ is large enough, then we can use Eq. 14. This is what is done in Figs. 5-8. The black circles, blue stars, and red triangles show the $p_{T}, p_{z}$ and $E$ terms, respectively, from the first-order expansion (Eq. 14), while the open circles and orange inverted triangles represent the results of Eq. 14 and Eq. 13, respectively.

Several observations are in order. Firstly, each of the three terms in Eq. 14 produce non-trivial behavior of the $A_{l, m}$ 's, interfering with each other in interesting ways. We find also that the $p_{z}$ term affects $A_{2,2}$; this was initially surprising since $A_{2,2}$ quantifies the behavior of the correlation function in the "outside" plane, while $\hat{z}$ is the "long" direction in the Bertsch-Pratt system. Clearly, EMCICs projected onto a 2-particle space are non-trivial objects.

It is seen that the first-order expansion (Eq. 14) agrees well with the full expression (Eq. 13) well for $N>\sim 10$. Such multiplicities are relevant for the $p+p$ measurements done at RHIC (especially recalling that $N$ includes all particles, even unmeasured ones). We see also that the analytic calculations (open circles and inverted triangles) approximate the results of the GENBOD simulation (green squares), especially as the multiplicity and total energy of the event increases; increasing agreement for large $N$ and $E_{t o t}$ is expected, given the approximations leading to our analytic expressions. We observe also that the analytically-calculated expressions respond identically to the kinematic cuts as does the simulation (c.f. Figs. 5 and 6).

Finally, the analytic calculations never reproduce exactly the simulations; we discuss this further in the next Section.

\section{AN EXPERIMENTALIST'S FORMULA}

Even for large $N$ and energy, the calculations do not exactly reproduce the EMCIC effects in the simulation. One reason for this may be found, in fact, in the definition of the average values (e.g. $\left\langle p_{z}^{2}\right\rangle$ ) themselves. In Eq. 11, average quantities are calculated using the distribution $\tilde{f}(p)$, which is not affected by EMCICs. Naturally, the only measurable distribution available to the experimentalist (even when GENBOD simulations serve as the "experiment") is $\tilde{f}_{c}(p)$.

Thus, it appears the experimentalist cannot plug her data into the equations 10, 11 and 14 to fully calculate EMCICs. However, such an ambition would have been hopeless anyhow. After all, even the total multiplicity $N$ (again, including photons etc) is rarely fully measured. And finite kinematic acceptance (e.g. in $\eta$ ) will require extrapolation to calculate, e.g. $\left\langle p_{z}^{2}\right\rangle$.

To the practicing femtoscopist, there is a natural solution. Having at hand (1) educated guesses for the quantities $N,\left\langle E^{2}\right\rangle$ etc, and (2) a physically-motivated functional form which connects these quantities to the correlations we'd like to un- 
derstand, we perform a fit. Let us rewrite Eq. 14 as

$$
\begin{gathered}
C\left(p_{1}, p_{2}\right)=1-M_{1} \cdot \overline{\left\{\vec{p}_{1, T} \cdot \vec{p}_{2, T}\right\}}-M_{2} \cdot \overline{\left\{p_{1, z} \cdot p_{2, z}\right\}} \\
-M_{3} \cdot \overline{\left\{E_{1} \cdot E_{2}\right\}}+M_{4} \cdot \overline{\left\{E_{1}+E_{2}\right\}}-\frac{M_{4}^{2}}{M_{3}} .
\end{gathered}
$$

where

$$
\begin{gathered}
M_{1} \equiv \frac{2}{N\left\langle p_{T}^{2}\right\rangle} \quad, \quad M_{2} \equiv \frac{1}{N\left\langle p_{z}^{2}\right\rangle} \\
M_{3} \equiv \frac{1}{N\left(\left\langle E^{2}\right\rangle-\langle E\rangle^{2}\right)} \quad, \quad M_{4} \equiv \frac{\langle E\rangle}{N\left(\left\langle E^{2}\right\rangle-\langle E\rangle^{2}\right)} .
\end{gathered}
$$

The notation $\overline{\{X\}}$ in Eq. 15 highlights the fact that $X$ is a two-particle quantity which depends on $p_{1}$ and $p_{2}$ (or $\vec{q}$, etc): $\overline{\{X\}}(\vec{q})$. From a practical point of view, $X$ is averaged over the same $\vec{q}$ bins as used for the correlation function. This involves nothing more than adding four more histograms to the several already being constructed by the experimentalist as she runs through all pairs in the data. The binned functions $\overline{\{X\}}$ then automatically reflect the same event and particle selection as the correlation function.

It is appropriate here to re-emphasize the point made in reference to Eq. 13. The event's total energy and momentum is a fixed quantity in a fixed (e.g. lab) frame; in particular, the momentum in Eq. 13 is assumed $\vec{P}=\overrightarrow{0}$ - i.e. the collision-centerof-mass (CCM) frame is assumed. In a pair-dependent frame (e.g. pair center-of-mass PCM or longitudinally co-moving system LCMS), the event's energy and momentum will depend on the pair. EMCICs, therefore, must be calculated with CCM momentum. Thus, in the function $\overline{\left\{p_{1, z} \cdot p_{2, z}\right\}}(\vec{q}), p_{1, z}$ and $p_{2, z}$ must be calculated in the CCM frame, while the binning variable $\vec{q}$ should be in whatever frame one chooses to construct the correlation function $C$.

The parameters $M_{i}$ defined in Eq. 16, on the other hand, are global and independent of $p_{1}$ and $p_{2}$. It is these which we will use as fit parameters. The task is then fast and straightforward; the EMCIC part of the correlation function $C(\vec{q})$ is simply a weighted sum of four functions. Indeed, one may calculate coefficients as in Eq. 1 for the four new functions. For example

$$
\begin{aligned}
A_{l, m}^{p_{Z}}(Q) \equiv \sum_{\text {bins } i} \overline{\left\{p_{1, z} \cdot p_{2, z}\right\}}\left(Q, \cos \theta_{i}, \phi_{i}\right) . \\
Y_{l, m}\left(\cos \theta_{i}, \phi_{i}\right) F_{l, m}\left(\cos \theta_{i}, \Delta_{\cos \theta}, \Delta_{\phi}\right),
\end{aligned}
$$

etc. Then, thanks to the linearity of Eq. 15 and the orthonormality of $Y_{l, m}$ 's, the measured $A_{l, m}$ 's themselves are similarly just weighted sums of harmonics

$$
\begin{aligned}
& A_{l, m}(Q)=\delta_{l, 0} \cdot\left(1-M_{4}^{2} / M_{3}\right)-M_{1} \cdot A_{l, m}^{p_{T}}(Q) \\
& -M_{2} \cdot A_{l, m}^{p_{Z}}(Q)-M_{3} \cdot A_{l, m}^{(E \cdot E)}(Q)+M_{4} \cdot A_{l, m}^{(E+E)}(Q) .
\end{aligned}
$$

Treating Eq. 18 as a fit, we have a few (say six, for $l \leq 4$ ) one-dimensional functions to fit with four adjustable weights.

A first example of such a fit is shown in Fig. 10. Again the GENBOD simulation is compared to the first-order form of Eq. 15. The filled circles, stars and triangles show the " $p_{T}$ "
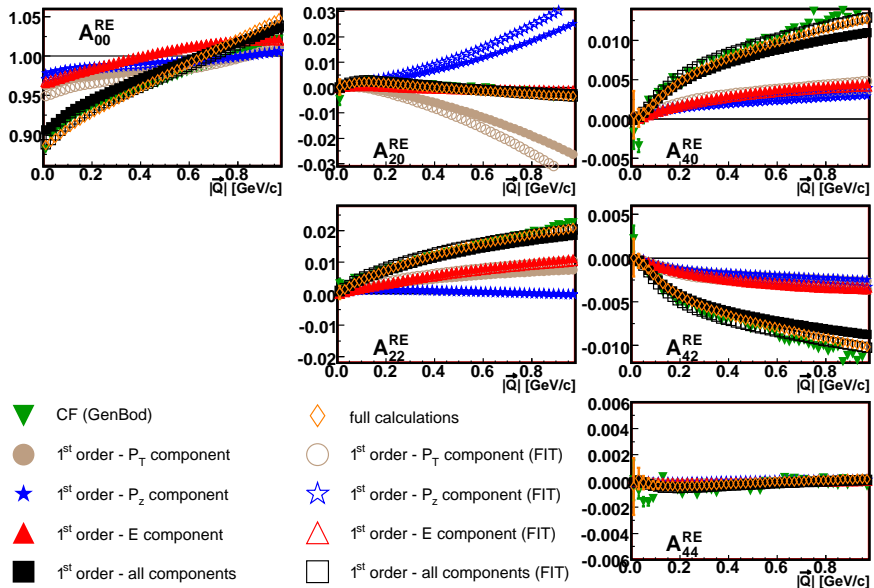

FIG. 10: (Color online) $A_{l, m}$ 's from 18-pion GENBOD -generated events. Green inverted triangles (often underneath black squares) is the correlation function (measured in PRF) from GENBOD . Filled brown circles, filled blue stars and filled red triangles show, respectively, the " $p_{T}$," " $p_{z}$," and " $E$ " terms, defined in Eq. 14; black filled squares show their sum. Open symbols of the same shape and color (identified as "FIT" in the legend) show corresponding terms, except with weights (see Eq. 16) adjusted to maximize agreement between the open black squares and the simulation.

$\left(M_{1}\right)$, " $p_{z}$ " $\left(M_{2}\right)$, and "E" $\left(M_{3}\right.$ and $\left.M_{4}\right)$ terms when the weights (Eq. 16) are calculated directly from the events, as discussed in Section IV. Treating the $M_{i}$ as adjustable parameters leads to a slightly different weighting of the terms, and a slightly better fit to the data.

Lest we forget, our original goal was not to understand EMCICs per se, but to extract the femtoscopic information from measured two-particle correlations. Assuming that the only non-femtoscopic correlations are EMCICs, one may simply add the femtoscopic terms (e.g. Gaussian in $\left(q_{o}, q_{s}, q_{l}\right)$ space or whatever) to the fitting function in Eq. 15 or 18 . Common femtoscopic fitting functions usually contain $\sim 5$ parameters (e.g. $R_{\text {out }}$ ) In the imaging technique [19], one assumes the separation distribution is described by a sum of splines (rather than, say, a Gaussian); here, too, there are usually 4-5 fit parameters (spline weights). We have found that the number of fit parameters now must be doubled to account also for EMCICs. This is a non-trivial increase in analysis complexity. However, we keep in mind two points.

Firstly, the increased work is necessary. EMCICs (and possibly other important non-femtoscopic correlations) are present and increasingly relevant at low multiplicity. One option is to ignore them, as has sometimes been done in early high-energy experiments. However, with the new high-quality data and desire for high-detail understanding at RHIC, ignoring obvious features such as those seen in Figs. 1 and 2 is clearly unacceptable. Perhaps a slightly better option is to invent an ad-hoc functional form with no real physical basis (and often manifestly wrong symmetry [cf 13]), which introduces new parameters in any case. We hope that the results here present a relatively painless, and considerably more responsible, third option. 
Secondly, while the non-femtoscopic EMCICs are not confined to the large- $Q$ region (an important point!), the femtoscopic correlations are confined to the small- $Q$ region. Therefore, one hopes that the addition of four new parameters to the fit of the correlation function will not render the fit overly unwieldy. While we can not expect complete blockdiagonalization of the fit covariance matrix, one hopes that the $M_{i}$ are determined well enough at high $Q$ that the femtoscopic fit parameters can be extracted at low $Q$.

\section{SUMMARY}

To truly claim an understanding of the bulk nature of matter at RHIC and the LHC, a detailed picture of the dynamicallygenerated geometric substructure of the system created in heavy ion collisions is needed. It is believed that this substructure, and the matter itself, is dominated by strong collective flow. The most direct measure of this flow is a measurement of the space-momentum correlation (e.g. $\left.R\left(m_{T}\right)\right)$ it generates. The physics of this large system, and the signals it generates, should be compared to the physics dominating $p+p$ collisions, as is increasingly common in high- $p_{T}$ studies at RHIC. For the small systems, however, non-femtoscopic effects contribute significantly to the correlation function, clouding the extraction and interpretation of the femtoscopic ones.

EMCICs, correlations generated by kinematic conservation laws, are surely present and increasingly relevant as the event multiplicity is reduced. Using the code GENBOD to study correlation functions solely driven by EMCICs, we found highly non-trivial structures strongly influenced by event characteristics (multiplicity and energy) and kinematic particle selection.

We extended the work of Danielewicz and Ollitrault to include four-momentum conservation and applied it to corre- lation functions commonly used in femtoscopy. We found structures associated individually with the conservation of the four-momentum components, which interfere in nontrivial ways. Comparison of the analytic EMCIC calculations with the GENBOD simulation gave confidence that the approximations (e.g. "large" multiplicity $N$ ) entering into the calculation were sufficiently valid, at least for multiplicities considered here. We further showed that the full EMCIC calculation can safely be replaced with a first-order expansion in $1 / N$.

Based on this first-order expansion, we developed a practical, straight-forward "experimentalist's formula" to generate histograms from the data which are later used in a generalized fit to the measured correlation function, including EMCICs and femtoscopic correlations.

The huge systematics of results and interest in femtoscopy in heavy ion collisions is renewing similar interest in the space-time signals from $p+p$ collisions. Direct comparisons between the two systems are now possible at RHIC and have already produced intriguing (albeit preliminary) results. Very soon, $p+p$ collisions will be measured in the LHC experiments, and the heavy ion experimentalists will be eager to apply their tools. The femtoscopic tool is one of the best in the box - so long as we keep it sufficiently calibrated with respect to non-femtoscopic effects increasingly relevant in small systems.

We would like to thank the organizers of this workshopmost especially the tireless Dr. Sandra Padula- for arranging a enjoyable gathering of experts in a very productive environment. We wish to thank Drs. Mark Baker, Nicolas Borghini, Ulrich Heinz, Adam Kisiel, Konstantin Mikhaylov, JeanYves Ollitrault, and Alexey Stavinsky for important suggestions and insightful discussions.
[1] Jean-Yves Ollitrault, Phys. Rev. D 46, 229 (1992).

[2] J. Adams et al., nucl-ex/0501009

[3] K. Adcox et al., nucl-ex/0410003.

[4] B. B. Back et al., nucl-ex/0410022.

[5] I. Arsene et al., nucl-ex/0410020.

[6] M.A. Lisa, S. Pratt, R. Soltz, and U. Wiedemann, Ann. Rev. Nucl. Part. Sci. 55, 311 (2005).

[7] S. Pratt, Phys. Rev. Lett. 53, 1219 (1984).

[8] F. Retiere and M.A. Lisa, Phys. Rev. C 70, 044907 (2004).

[9] Gideon Alexander, Rept. Prog. Phys. 66, 481 (2003).

[10] W. Kittel and E. A. De Wolf, p. 652 (Hackensack, USA: World Scientific, 2005)

[11] Z. Chajecki, Nucl. Phys. A 774, 599 (2006).

[12] Z. Chajecki, T. D. Gutierrez, M. A. Lisa, and M. LopezNoriega, nucl-ex/0505009.

[13] Z. Chajecki, AIP Conf. Proc. 828, 566 (2006).

[14] F. James, CERN-68-15.

[15] P. Danielewicz et al., Phys. Rev. C 38, 120 (1988).

[16] Nicolas Borghini, Phuong Mai Dinh, and Jean-Yves Ollitrault,
Phys. Rev. C 62, 034902 (2000).

[17] Nicolas Borghini, Eur. Phys. J. C 30, 381 (2003).

[18] Nicolas Borghini, nucl-th/0612093.

[19] D.A. Brown and P. Danielewicz, Phys. Lett. B 398, 252 (1997).

[20] As an example, $p_{z}$ conservation should not affect observables in the transverse plane (e.g. elliptic flow $v_{2}$ ), since $p_{z}$ is independent of $p_{x y}$. On the other hand, energy conservation, in the $D=3 \oplus 1$ case, can in principle affect $v_{2}$.

[21] Our use of symbols $f$ and $f_{c}$ follows the convention used in [16], which is significantly different - if unfortunately similar-looking - than that used in [17] and [18].

[22] The authors are grateful to U. Heinz for showing that on-shell conditions do not cause major complications in the extension to the formula to include energy conservation.

[23] In principle, also required for Eq. 9 is $\langle E\rangle^{2} \gg\left\langle E^{2}\right\rangle$ so that negative energies have negligible probability. This requirement may be significantly violated in reality. 